Article

\title{
Improved Performance of GaN-Based Light-Emitting Diodes Grown on Si (111) Substrates with $\mathrm{NH}_{3}$ Growth Interruption
}

\author{
Sang-Jo Kim ${ }^{1,+}{ }^{\mathbb{D}}$, Semi Oh ${ }^{2, \dagger}$, Kwang-Jae Lee ${ }^{3} \mathbb{D}$, Sohyeon $\mathrm{Kim}^{4}$ and Kyoung-Kook Kim ${ }^{4, *}$ \\ 1 School of Materials Science and Engineering, Gwangju Institute of Science and Technology, \\ Gwangju 61005, Korea; prokimsj@gmail.com \\ 2 Department of Electrical Engineering and Computer Science, University of Michigan, \\ Ann Arbor, MI 48109, USA; ohsemi1230@gmail.com \\ 3 Department of Electrical Engineering, Stanford University, Stanford, CA 94305, USA; kwangjae@stanford.edu \\ 4 Department of Advanced Convergence Technology, Research Institute of Advanced Convergence Technology, \\ Korea Polytechnic University, 237 Sangidaehak-ro, Siheung-si 15073, Korea; sohyeon.kim@kpu.ac.kr \\ * Correspondence: kim.kk@kpu.ac.kr \\ + These authors equally contributed.
}

Citation: Kim, S.-J.; Oh, S.; Lee, K.-J.; Kim, S.; Kim, K.-K. Improved

Performance of GaN-Based

Light-Emitting Diodes Grown on $\mathrm{Si}$ (111) Substrates with $\mathrm{NH}_{3}$ Growth Interruption. Micromachines 2021, 12, 399. https://doi.org/10.3390/ mi12040399

Academic Editor: Giovanni Verzellesi

Received: 10 March 2021

Accepted: 2 April 2021

Published: 5 April 2021

Publisher's Note: MDPI stays neutral with regard to jurisdictional claims in published maps and institutional affiliations.

Copyright: (c) 2021 by the authors. Licensee MDPI, Basel, Switzerland. This article is an open access article distributed under the terms and conditions of the Creative Commons Attribution (CC BY) license (https:/ / creativecommons.org/licenses/by/ $4.0 /)$.

\begin{abstract}
We demonstrate the highly efficient, GaN-based, multiple-quantum-well light-emitting diodes (LEDs) grown on $\mathrm{Si}$ (111) substrates embedded with the AlN buffer layer using $\mathrm{NH}_{3}$ growth interruption. Analysis of the materials by the X-ray diffraction omega scan and transmission electron microscopy revealed a remarkable improvement in the crystalline quality of the GaN layer with the AlN buffer layer using $\mathrm{NH}_{3}$ growth interruption. This improvement originated from the decreased dislocation densities and coalescence-related defects of the $\mathrm{GaN}$ layer that arose from the increased $\mathrm{Al}$ migration time. The photoluminescence peak positions and Raman spectra indicate that the internal tensile strain of the GaN layer is effectively relaxed without generating cracks. The LEDs embedded with an AlN buffer layer using $\mathrm{NH}_{3}$ growth interruption at $300 \mathrm{~mA}$ exhibited $40.9 \%$ higher light output power than that of the reference LED embedded with the AlN buffer layer without $\mathrm{NH}_{3}$ growth interruption. These high performances are attributed to an increased radiative recombination rate owing to the low defect density and strain relaxation in the GaN epilayer.
\end{abstract}

Keywords: AlN buffer layer; $\mathrm{NH}_{3}$ growth interruption; strain relaxation; GaN-based LED; low defect density

\section{Introduction}

Substantial progress in fabricating highly efficient, GaN-based, multiple-quantumwell (MQW) light-emitting diodes (LEDs) has proven these materials useful in a variety of applications, such as micro-displays, automobiles, general lighting, and optoelectronics [1-3]. Furthermore, GaN-based epitaxial-layers for LED devices are conventionally fabricated on sapphire and $\mathrm{SiC}$ substrates. However, the sapphire substrate has poor thermal conductivity-as low as $25 \mathrm{Wm}^{-1} \mathrm{~K}^{-1}$ - that encourages heat dissipation. Therefore, it is a critical issue in high-output LED operation. Additionally, $\mathrm{GaN}$ and $\mathrm{SiC}$ substrates are expensive and still limitedly used in large-scale LED fabrication than a sapphire substrate $[4,5]$.

The large-scale Si substrate has attracted significant interest in growing GaN-based devices owing to lower cost than the traditional sapphire and $\mathrm{SiC}$ substrates [6,7]. Moreover, Si substrate can be easily integrated with electronic and optical devices [8].

However, growing a high-quality GaN epitaxial layer on a $\mathrm{Si}$ (111) substrate presents several key challenges. First, when the GaN layer is grown directly on the Si substrate, the Si surface easily reacts with $\mathrm{NH}_{3}$ to form the $\mathrm{SiN}_{\mathrm{x}}$, which the GaN layer cannot grow. Subsequently, the Si substrate reacts with $\mathrm{Ga}$ to promote melt-back etching of Ga-Si eutectic alloys [9]. Second, the large lattice mismatch ( 17\%) between $\mathrm{GaN}$ and $\mathrm{Si}$ (111) causes a 
high dislocation density in the GaN layer leading to lower LED performance [10]. Third, the difference in thermal expansion coefficients $(\sim 56 \%)$ between $\mathrm{Si}$ and $\mathrm{GaN}$ introduces large tensile stress in the GaN layer during the cooling process from the high growth temperature, which causes the wafer bowing and cracks generation [11].

Therefore, many researchers have introduced various methods to reduce threading dislocation, stress mitigation, and remove cracks, such as epitaxial lateral overgrowth, nanoporous $\mathrm{GaN}$ layers, graded $\mathrm{AlGaN}$ interlayers, and $\mathrm{Al}(\mathrm{Ga}) \mathrm{N} / \mathrm{GaN}$ superlattices [12-15]. Particularly, the AlN layer, which acts as a bottom buffer layer on the Si substrate, significantly affects crystalline quality and stress management of the GaN layer.

AlN layers with rough surfaces and poor crystalline qualities lead to GaN layers with poor crystalline quality. Therefore, many studies have revealed that high-quality AlN buffer layers minimize crystal misorientations and dislocation density in the GaN layer. Krost et al. developed a low-temperature (LT)-AlN layer-that is, a novel method to reduce stress [16].

Comparatively, high-temperature (HT)-AlN layers yield reduced dislocation densities and promote the relaxation of compressive strain due to an increased $\mathrm{Al}$ migration length. In addition, many studies are also investigated the high-quality GaN layer grown on a Si substrate using the HT- and LT-AlN growth process $[17,18]$. Moreover, Hirayama et al. used an AlN buffer layer grown with pulsed $\mathrm{NH}_{3}$ flow on a sapphire substrate [19], which is an effective way to enhance the lateral migration of $\mathrm{Al}$ atoms and produce a smooth epitaxial surface.

However, there have been no reports on the effects of ammonia $\left(\mathrm{NH}_{3}\right)$ growth interruption for the AlN layer in GaN-based LEDs grown on $\mathrm{Si}$ (111) substrates.

We demonstrate that AlN layers prepared with $\mathrm{NH}_{3}$ growth interruption to be served as a buffer layer improve the crystalline quality and strain relaxation in GaN-based LEDs grown on $\mathrm{Si}$ (111) substrates. The $\mathrm{Al}$ migration time of the $\mathrm{AlN}$ was controlled by $\mathrm{NH}_{3}$ pulse timing.

The optical output power of LEDs grown using $\mathrm{NH}_{3}$ growth interruption was $40.9 \%$ greater than that of the reference LED embedded with the AlN buffer layer without $\mathrm{NH}_{3}$ growth interruption (injection current of $300 \mathrm{~mA}$ ). Such remarkable improvements of optical output power are predominantly attributed to reduced dislocation densities and strain relaxation, which originated from an increased Al migration in the AlN buffer layer.

\section{Materials and Methods}

InGaN/GaN MQW LEDs were grown on Si (111) substrates using metal-organic chemical vapor deposition. Trimethylaluminum (TMAl) and $\mathrm{NH}_{3}$ were used as the $\mathrm{Al}$ and $\mathrm{N}$ sources, respectively, and high-purity hydrogen was employed as the carrier gas. $\mathrm{Si}$ (111) substrates were first annealed at $1050{ }^{\circ} \mathrm{C}$ for $10 \mathrm{~min}$ to remove the native oxide. The substrates were subsequently passivated by TMAl with a pre-dose time of $10 \mathrm{~s}$ to prevent Si melt-back etching. A $200 \mathrm{~nm}$-thick AlN layer was subsequently deposited at $1050{ }^{\circ} \mathrm{C}$ as a reference. Comparatively, for samples prepared by $\mathrm{NH}_{3}$ growth interruption, TMAl was constantly introduced into the chamber while $\mathrm{NH}_{3}$ was injected into the reactor following a specified pulsed regimen. Specifically, the halted $\left(t_{1}\right) \mathrm{NH}_{3}$ flow time was changed: $3 \mathrm{~s}$ for sample A, $5 \mathrm{~s}$ for sample B, $7 \mathrm{~s}$ for sample $\mathrm{C}$, and $9 \mathrm{~s}$ for sample $\mathrm{D}$, as schematically shown in Figure 1.

After that, to prevent crack formation, two pairs of $12 \mathrm{~nm}$-thick low-temperature AlN layers and a $1.2 \mu \mathrm{m}$-thick undoped $\mathrm{GaN}$ layer were deposited.

Furthermore, a $1.5 \mu \mathrm{m}$-thick $n$-type GaN layer $\left(n=6 \times 10^{18} / \mathrm{cm}^{3}\right)$, six periods of $2.3 \mathrm{~nm}$ thick $\operatorname{In}_{0.18} \mathrm{Ga}_{0.82} \mathrm{~N}$ layer, and $7.7 \mathrm{~nm}$-thick GaN-based MQWs layers were subsequently grown. The $15 \mathrm{~nm}$-thick $\mathrm{Al}_{0.15} \mathrm{Ga}_{0.85} \mathrm{~N}$ electron blocking layer (EBL) and the $200 \mathrm{~nm}$-thick $p$-type GaN layer $\left(\left(n=5 \times 10^{19} / \mathrm{cm}^{3}\right)\right.$ were finally deposited. 
To fabricate the n-electrode, the epilayers were partially etched until the $n$-type GaN layer was exposed. The $200 \mathrm{~nm}$-thick ITO layer was deposited using an electron-beam evaporator on the remaining parts of the p-type $\mathrm{GaN}$ layer and annealed at $600{ }^{\circ} \mathrm{C}$ in $\mathrm{O}_{2}$ atmosphere for $1 \mathrm{~min}$ using the rapid thermal annealing. The Ti/ $\mathrm{Al}(50 / 200 \mathrm{~nm})$ layers were deposited as an n-electrode. Finally, the $\mathrm{Cr} / \mathrm{Al}(30 / 200 \mathrm{~nm})$ layers were deposited on the $p$ - and $n$-electrodes and annealed at $300{ }^{\circ} \mathrm{C}$ for $1 \mathrm{~min}$.

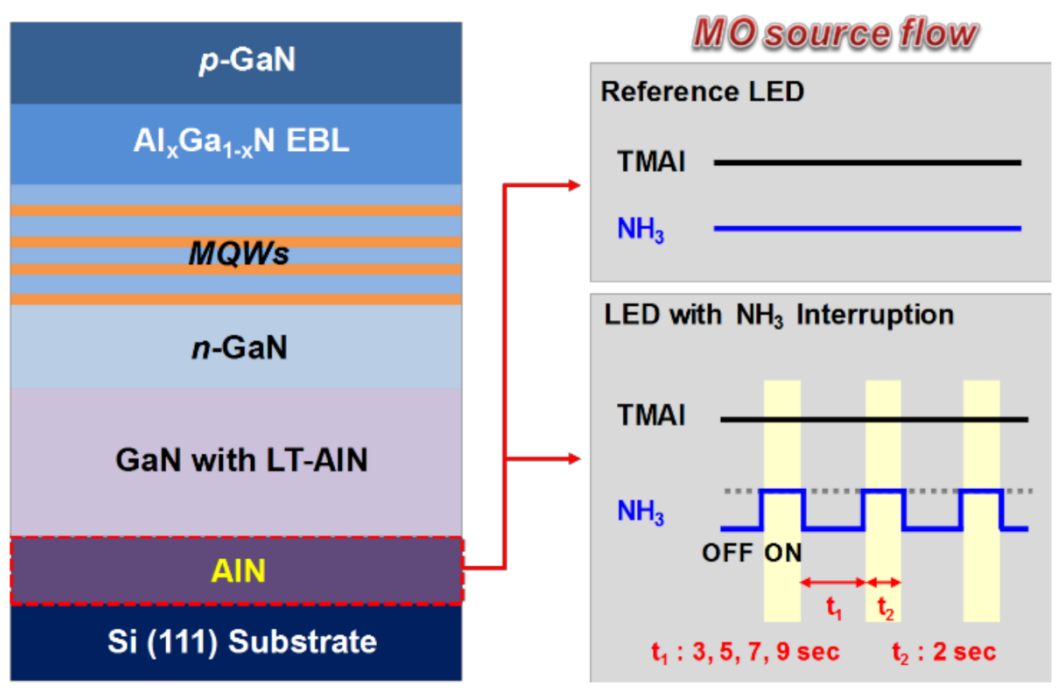

Figure 1. Schematics of light-emitting diodes (LEDs), including the sequence of all growth steps prepared with and without the $\mathrm{NH}_{3}$ growth interruption method.

\section{Results and Discussion}

\subsection{Epitaxial Characteristics}

The effect of $\mathrm{NH}_{3}$ growth interruption on the crystal quality of $\mathrm{GaN}$ was explored using X-ray diffraction (XRD) (PANalytical X'Pert PRO, Almelo, Netherlands) (reference sample and Sample C), as shown in Figure 2a,b. In addition, all samples of full-width-athalf-maximum (FWHM) values of the GaN (0002), GaN (10-12), and AlN (0002) reflections are plotted in Figure 3a.
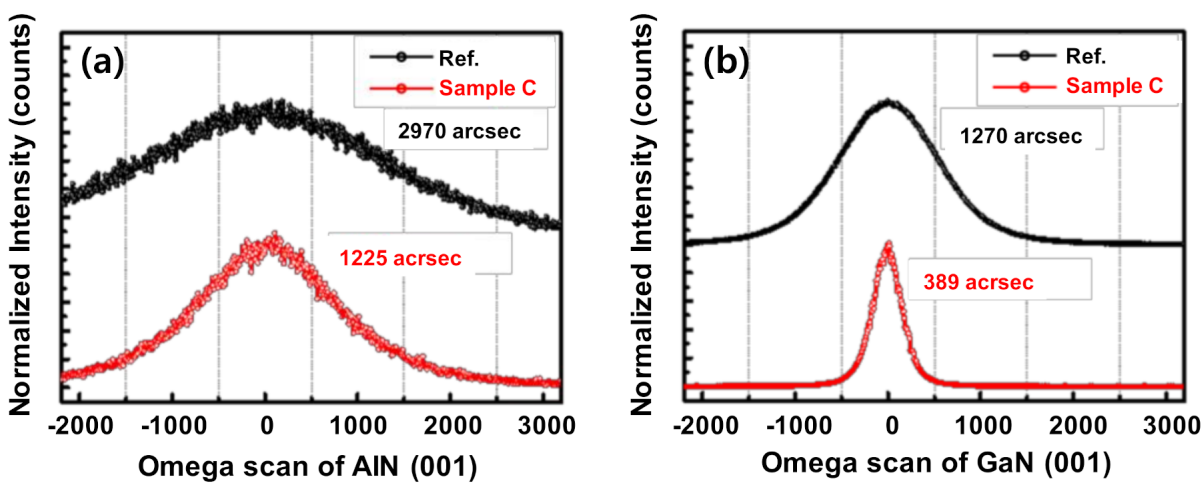

Figure 2. X-ray diffraction (XRD) Omega scan of reference and sample C (a) AlN (001) and (b) GaN (001). 
(a)

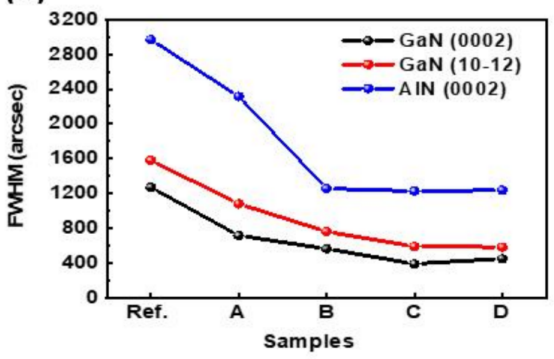

(d)

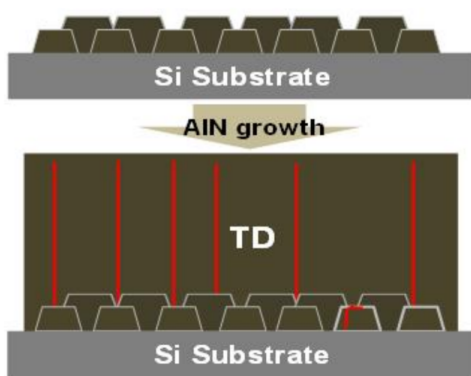

(b)

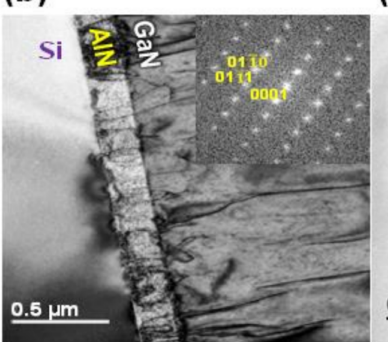

(c)
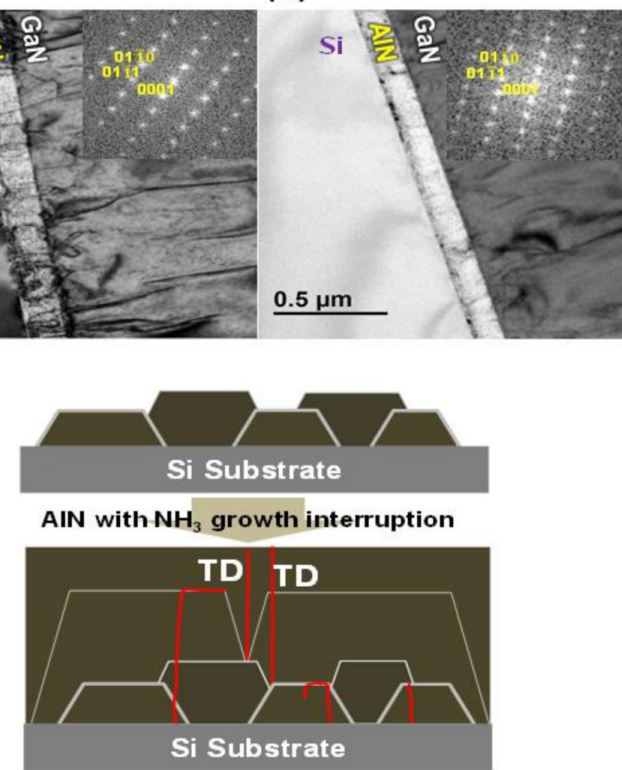

Figure 3. (a) The full-width-at-half-maximum (FWHM) values of the GaN (0002), GaN (10-12), and AlN (0002) reflections for all the samples. TEM images of (b) a reference sample and (c) a sample C. (d) Schematic growth mechanism of AlN with and without the $\mathrm{NH}_{3}$ growth interruption.

The full-width-at-half-maximum (FWHM) of the (0002) and (10-12) reflections of GaN and AIN layers typically indicate imperfections in the crystal showing the densities of the screw $\left(D_{s}\right)$ and edge $\left(D_{e}\right)$ dislocations in the epitaxial layer, respectively. The parameters $D_{s}$ and $D_{e}$ can be obtained using the following equations $[20,21]$ :

$$
\begin{aligned}
& D_{S}=\beta_{(0002)}^{2} / 4.35 b_{c}^{2} \\
& D_{e}=\beta_{(10-12)}^{2} / 4.35 b_{a}^{2}
\end{aligned}
$$

where $\beta^{2}{ }_{(0002)}$ and $\beta^{2}{ }_{(10-12)}$ denote the FWHM of the (0002) and (10-12) reflections, respectively; $b_{c}$ and $b_{a}$ represent the Burgers vector lengths of the $c$ - and $a$-axial lattice constant, respectively. The FWHM values of the (0002) and (10-12) reflections of GaN were 714 and 1079 arcsec for sample A, 563.4 and 760.3 arcsec for sample B, and 389 and 589.3 arcsec for sample $C$, respectively. These FWHM values are much lower than the corresponding FWHM values (1270 and $1580 \mathrm{arcsec})$ of a reference sample. The corresponding $D_{\mathrm{s}}$ and $D_{\mathrm{e}}$ are tabulated in Table 1 . These results contain similar values to the other papers mentioned in introduction of HT-AlN, and LT-AlN growth for high-quality GaN layer grown on a Si substrate $[17,18]$.

Table 1. The full-width-at-half-maximum (FWHM) values of the X-ray diffraction (XRD) rocking curve and calculated dislocation densities for all the samples.

\begin{tabular}{cccccccc}
\hline \multirow{2}{*}{ Sample } & \multirow{2}{*}{$\boldsymbol{t}_{\mathbf{1}} \boldsymbol{t}_{\mathbf{2}}$} & \multicolumn{3}{c}{ XRD FWHM (arcsec) } & \multicolumn{2}{c}{ Dislocation Density $\left(\times \mathbf{1 0}^{\mathbf{9}} \mathbf{c m}^{-\mathbf{2}}\right)$} \\
\cline { 3 - 8 } & & GaN (001) & GaN (102) & AlN (001) & $\boldsymbol{D}_{\boldsymbol{s}}$ (GaN) & $\boldsymbol{D}_{\boldsymbol{e}}(\mathbf{G a N})$ & $\boldsymbol{D}_{\boldsymbol{s}}(\mathbf{A l N})$ \\
\hline Ref. & $t_{1}$ & 1270 & 1580 & 2970 & 13.8 & 56 & 81.7 \\
Sample A & $3 / 2$ & 714 & 1079 & 2316 & 4.36 & 26.3 & 49.7 \\
Sample B & $5 / 2$ & 563 & 760 & 1255 & 2.71 & 13 & 14.6 \\
Sample C & $7 / 2$ & 389 & 589 & 1225 & 1.29 & 7.85 & 13.9 \\
Sample D & $9 / 2$ & 448 & 581 & 1237 & 1.71 & 7.63 & 14.2 \\
\hline
\end{tabular}


$\mathrm{Al}$ adatoms have a high sticking coefficient and short migration time on Si substrates; therefore, they have a significantly low probability of moving from their point of impact. This gives rise to many nucleation sites for growth with a high density of extended defects, such as dislocations and grain boundaries [22]. Moreover, growth interruption by halting $\mathrm{NH}_{3}$ gas flow provides $\mathrm{Al}$ adatoms with sufficient residence time to incorporate the most energetic favorable lattice sites on the Si substrate. It promotes a large number of small nucleation sites, which further minimizes coalescence-related defects. However, significantly long migration time for sample D promotes AlN layers with lower crystalline quality and increased dislocation densities compared to those of sample $\mathrm{C}$ due to the deteriorating surface roughness, as shown in Table 1 [23-25].

We analyzed transmission electron microscopy (TEM) to deeply investigate the crystalline quality of AlN layers produced using $\mathrm{NH}_{3}$ growth interruption. Figure $3 b, c$ show the cross-sectional bright-field TEM images of the reference sample and sample $\mathrm{C}$, respectively, which were acquired near the GaN [0001] zone axis. Dislocations were clearly observed at the interface of the AlN and $\mathrm{Si}$ in the reference material; however, they are remarkably reduced in sample $\mathrm{C}$ because the longer $\mathrm{Al}$ migration time (as shown in Figure $3 \mathrm{~d}$ ) decreases coalescence related defects and dislocation densities.

The impact of the $\mathrm{NH}_{3}$ growth interruption process on the residual stress in the $\mathrm{GaN}$ epilayer was quantified by photoluminescence (PL) and Raman measurements, as shown in Figure 4a,b, respectively. The PL intensities of samples prepared by the $\mathrm{NH}_{3}$ growth interruption process increased with $\mathrm{Al}$ migration time $\left(t_{1}\right)$, indicating higher $\mathrm{GaN}$ crystal quality than the other samples. However, the PL intensity decreased with longer Al migration times. Moreover, the PL peak position of a sample $C$ blue-shifted from $366.7 \mathrm{~nm}$ to $365.3 \mathrm{~nm}$ and subsequently red-shifted to $365.6 \mathrm{~nm}$ in sample $\mathrm{D}$, indicating that the residual tensile strain in sample $C$ is smaller than that in the other samples. Figure $4 \mathrm{c}$ shows the Raman spectra acquired from the reference sample and sample $C$, specifically using a $514 \mathrm{~nm}$ light with the excitation power of $2.4 \mathrm{~mW}$.

The $E_{2}$ (high) vibrational mode is highly sensitive to strain. Therefore, it is widely used to quantify the stress in $\mathrm{GaN}$ epilayers [25]. The wavenumber of the $E_{2}$ (high) mode of the reference sample and sample $C$ was $566.03 \mathrm{~cm}^{-1}$ and $567.18 \mathrm{~cm}^{-1}$, respectively. Both the values were red-shifted from the $E_{2}$ (high) wavenumber from a standard free-standing bulk GaN $\left(567.5 \mathrm{~cm}^{-1}\right)$, proving that the $\mathrm{GaN}$ epilayers in the reference and sample $\mathrm{C}$ were under tensile stress [26]. Shifts of the $E_{2}$ (high) phonon peak are related to the relaxation of residual strain; it can be calculated using the following equation [27]:

$$
\Delta \omega_{\gamma}-\omega_{0}=K_{\gamma} \cdot \sigma_{x x}
$$

where $\omega_{\gamma}$ and $\omega_{0}$ represent the Raman wavenumbers of the $E_{2}$ (high) phonon peak of sample $\mathrm{C}$ and reference sample, respectively. A proportionality factor $K_{\gamma}\left(4.2 \mathrm{~cm}^{-1} \mathrm{GPa}^{-1}\right)$ originates from hexagonal GaN [28]. The $E_{2}$ (high) phonon peak of sample $C$ was blueshifted by $1.15 \mathrm{~cm}^{-1}$ from that of the reference sample, corresponding to the impressive different relaxation of tensile stress $\left(\sigma_{x x}\right)(0.274 \mathrm{GPa})$. The relaxation of tensile strain in $\mathrm{GaN}$ layer for sample $\mathrm{C}$ causes the shrinking the lattice constant, which shifts the PL peak position to higher energy [29]. The reduced tensile stress in sample $C$ arises predominantly from the coalescence of large-sized grains due to the $\mathrm{NH}_{3}$ growth interruption method $[30,31]$. 

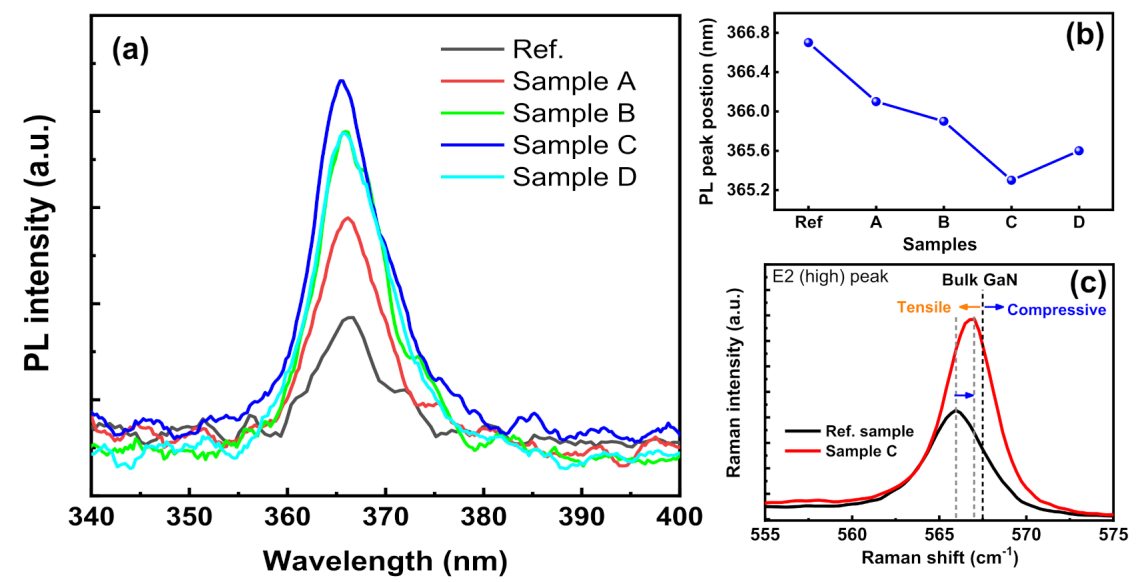

Figure 4. (a) Photoluminescence (PL) intensity and (b) PL peak position of all the studied samples. (c) Raman spectra of the reference sample and sample $\mathrm{C}$.

\subsection{Device Characteristics}

Figure 5 shows the current-voltage (I-V) characteristics and light output power of the reference sample and sample $C$. Figure 5 a reveals that the forward voltage of sample $C$ at an injection current of $20 \mathrm{~mA}$ was $3.76 \mathrm{~V}$, which is lower than that of the reference sample $(3.91 \mathrm{~V})$ under the same condition.

Furthermore, the series resistances estimated from the I-V curves of the reference sample and sample $C$ were $37.2 \mathrm{ohm}$ and $31.6 \mathrm{ohm}$, respectively. These values indicate that decreasing the density of defects, such as threading dislocations by $\mathrm{NH}_{3}$ growth interruption method, improves the crystal quality of GaN epi-layer; moreover, it results in a decreased series resistance and forward voltage. Figure $5 \mathrm{~b}$ shows that the light output power of sample $\mathrm{C}$ at an injection current of $300 \mathrm{~mA}$ is $40.9 \%$ greater than that of the reference sample; it is attributed to enhanced radiative recombination due to the reduced dislocation densities and relaxation of internal tensile strain. We could not measure the light output power above $300 \mathrm{~mA}$ by the limitation of measurement system. However, normally, as the injection current is increased, the carrier overflow and Auger recombination are also increased. Therefore, at high current, the portion of increasing light output power will be decreased $[32,33]$.
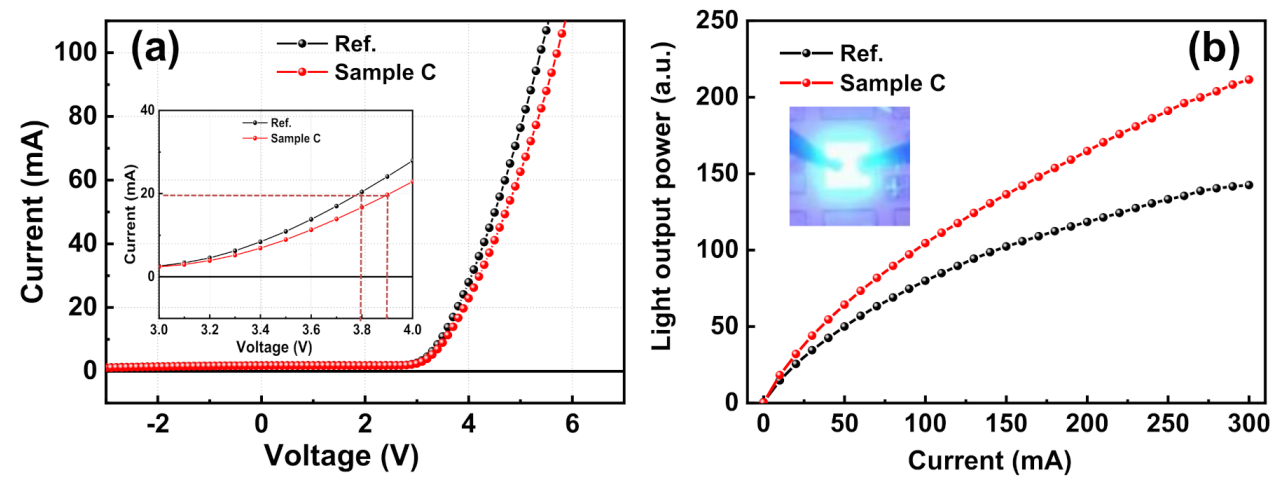

Figure 5. (a) Current-voltage (I-V) characteristics and (b) optical output power of the reference sample and a sample $\mathrm{C}$.

\section{Conclusions}

We demonstrated the $\mathrm{NH}_{3}$ growth-interruption process to prepare the high-performance InGaN/GaN MQW LEDs grown on Si (111) substrates. The XRD results revealed low FWHM values for GaN grown on AlN layers using the $\mathrm{NH}_{3}$ growth interruption method; it established substantially improved crystal quality compared to the reference LED embedded with the AlN buffer layer without $\mathrm{NH}_{3}$ growth interruption. 
Improved crystalline quality is further corroborated by comparative TEM analyses, which is attributed to the effectively reduced dislocation densities and coalescence by longer Al migration times. A lower forward voltage of $3.76 \mathrm{~V}$ was observed at an injection current of $20 \mathrm{~mA}$ for the LED fabricated by the $\mathrm{NH}_{3}$ growth interruption method; however, the reference LED had a forward voltage of $3.91 \mathrm{~V}$. The optical output power of the LED prepared using the $\mathrm{NH}_{3}$ growth interruption was $40.9 \%$ greater than that of the reference LED.

Such enhanced optical output is attributed to increased radiative recombination rates due to the decreased dislocation densities and relaxation of internal tensile strain, which arise from the longer $\mathrm{Al}$ migration time on the $\mathrm{Si}$ (111) substrate. These results demonstrate that the $\mathrm{NH}_{3}$ growth interruption method is an important technique for growing highperformance LEDs grown on $\mathrm{Si}$ (111) substrate. Therefore, these results ultimately represent a step towards realizing high-efficiency and high-power LEDs.

Author Contributions: Conceptualization, S.-J.K. and S.O.; methodology, S.-J.K. and K.J.L.; formal analysis, S.-J.K., K.J.L. and S.K; writing—original draft preparation, S.-J.K. and S.O.; writing-review and editing, S.O and K.-K.K.; supervision, K.-K.K. All authors have read and agreed to the published version of the manuscript.

Funding: This research was supported by the Ministry of Science and ICT (MIST), Korea, under the Information Technology Research Center (ITRC) support program (IITP-2020-2018-0-01426) supervised by the Institute for Information and Communications Technology Planning and Evaluation (IITP) by the Korea Institute for Advancement of Technology (KIAT) grant funded by the Korea Government (MOTIE) (P0008458, The Competency Development Program for Industry), and by the Technology Innovation Program (or Industrial Strategic Technology Development Program) (20002694, Gas sensor) funded by the Ministry of Trade, Industry, and Energy (MOTIE, Korea).

Conflicts of Interest: The authors declare no conflict of interest.

\section{References}

1. Schubert, E.F.; Kim, J.K. Solid-State Light Sources Getting Smart. Science 2005, 308, 1274-1278. [CrossRef] [PubMed]

2. Ponce, F.A.; Bour, D.P. Nitride-based semiconductors for blue and green light-emitting devices. Nature 1997, 386, 351-359. [CrossRef]

3. Rogers, J.A.; Someya, T.; Huang, Y. Materials and Mechanics for Stretchable Electronics. Science 2010, 327, 1603-1607. [CrossRef] [PubMed]

4. Liu, X.; Wang, H.Y.; Chiu, H.C.; Chen, Y.; Li, D.; Huang, C.R.; Kao, H.L.; Kuo, H.C.; Chen, S.W.H. Analysis of the back-barrier effect in AlGaN/GaN high electron mobility transistor on free-standing GaN substrates. J. Alloys Compd. 2020, $814,152293$. [CrossRef]

5. Li, C.; Li, Z.; Peng, D.; Yang, Q.; Zhang, D.; Luo, W.; Pan, C. Growth of thin AlN nucleation layer and its impact on GaN-on-SiC heteroepitaxy. J. Alloys Compd. 2020, 838, 155557. [CrossRef]

6. Zhang, X.; Li, P.; Zou, X.; Jiang, J.; Yuen, S.H.; Tang, C.W.; Lau, K.M. Active Matrix Monolithic LED Micro-Display Using GaN-on-Si Epilayers. IEEE Photonics Technol. Lett. 2019, 31, 865-868. [CrossRef]

7. Sun, Y.; Zhou, K.; Feng, M.; Li, Z.; Zhou, Y.; Sun, Q.; Liu, J.; Zhang, L.; Li, D.; Sun, X.; et al. Room-temperature continuous-wave electrically pumped InGaN/GaN quantum well blue laser diode directly grown on Si. Light Sci. Appl. 2018, 7, 13. [CrossRef]

8. Lee, K.H.; Bao, S.; Zhang, L.; Kohen, D.; Fitzgerald, E.; Tan, C.S. Integration of GaAs, GaN, and Si-CMOS on a common 200 mm Si substrate through multilayer transfer process. Appl. Phys. Express 2016, 9, 086501. [CrossRef]

9. Khoury, M.; Tottereau, Q.; Feuillet, G.; Vennegues, P.; Zuniga-Perez, J. Evolution and prevention of meltback etching: Case study of semipolar GaN growth on patterned silicon substrates. J. Appl. Phys. 2017, 122, 105108. [CrossRef]

10. Tran, C.A.; Osinski, A.; Karlicek, R.F.J. Growth of InGaN/GaN multiple-quantum-well blue light-emitting diodes on silicon by metalorganic vapor phase epitaxy. Appl. Phys. Lett. 1999, 75, 1494-1496. [CrossRef]

11. Dadgar, A.; Blasing, J.; Diez, A.; Alam, A.; Heuken, M.; Krost, A. Metalorganic Chemical Vapor Phase Epitaxy of Crack-Free GaN on Si (111) Exceeding $1 \mu \mathrm{m}$ in Thickness. Jpn. J. Appl. Phys. 2000, 39, L1183-L1185. [CrossRef]

12. Kim, B.; Lee, K.; Jang, S.; Jhin, J.; Lee, S.; Baek, J.; Yu, Y.; Lee, J.; Byun, D. Epitaxial Lateral Overgrowth of GaN on Si (111) Substrates Using High-Dose, N+ Ion Implantation. Chem. Vap. Depos. 2010, 16, 80-84. [CrossRef]

13. Lee, K.J.; Chun, J.; Kim, S.J.; Ha, C.S.; Park, J.W.; Lee, S.J.; Song, J.C.; Baek, J.H.; Park, S.J. Enhanced optical output power of InGaN/GaN light-emitting diodes grown on a silicon (111) substrate with a nanoporous GaN layer. Opt. Express 2016, 24, 4391-4398. [CrossRef]

14. Able, A.; Wegscheider, W.; Engl, K.; Zweck, J. Growth of crack-free GaN on Si (1 11 1) with graded AlGaN buffer layers. J. Cryst. Growth 2005, 276, 415-418. [CrossRef] 
15. Ni, Y.; He, Z.; Yang, F.; Zhou, D.; Yao, Y.; Zhou, G.; Shen, Z.; Zhong, J.; Zhen, Y.; We, Z.; et al. Effect of AlN/GaN superlattice buffer on the strain state in GaN-on-Si (111) system. Jpn. J. Appl. Phys. 2015, 54, 015505. [CrossRef]

16. Reiher, A.; Blasing, J.; Dadgar, A.; Diez, A.; Krost, A. Efficient stress relief in GaN heteroepitaxy on Si (111) using low-temperature AlN interlayers. J. Cryst. Growth. 2003, 248, 563-567. [CrossRef]

17. Lin, P.; Tien, C.; Wang, T.; Chen, C.; Ou, S.; Chung, B.; Wuu, D. On the role of AlN insertion layer in stress control of GaN on 150-mm Si (111) substrate. Crystals 2017, 7, 134. [CrossRef]

18. Lin, Y.; Yang, M.; Wang, W.; Lin, Z.; Li, G. A low-temperature AlN interlayer to improve the quality of GaN epitaxial films grown on Si substrates. CrystEngComm 2016, 18, 8926. [CrossRef]

19. Hirayama, H.; Yatabe, T.; Noguchi, N.; Ohashi, T.; Kamata, N. 231-261 nm AlGaN deep-ultraviolet light-emitting diodes fabricated on AlN multilayer buffers grown by ammonia pulse-flow method on sapphire. Appl. Phys. Lett. 2007, $91,071901$. [CrossRef]

20. Kaganer, V.M.; Brandt, O.; Trampert, A.; Ploog, K.H. X-ray diffraction peak profiles from threading dislocations in GaN epitaxial films. Phys. Rev. B. 2005, 72, 045423. [CrossRef]

21. Vickers, M.E.; Kappers, M.J.; Datta, R.; McAleese, C.; Smeeton, T.M.; Rayment, F.D.; Humphreys, C.J. In-plane imperfections in GaN studied by X-ray diffraction. J. Phys. D Appl. Phys. 2005, 38, A99-A104. [CrossRef]

22. Jain, R.; Sun, W.; Yang, J.; Shatalov, M.; Hu, X.; Sattu, A.; Lunev, A.; Deng, J.; Shturm, I.; Bilenko, Y.; et al. Migration enhanced lateral epitaxial overgrowth of AlN and AlGaN for high reliability deep ultraviolet light emitting diodes. Appl. Phys. Lett. 2008, 93, 051113. [CrossRef]

23. Rahman, M.N.A.; Talik, N.A.; Abdul, M.I.M.; Sulaiman, A.F.; Allif, K.; Zahir, N.M.; Shuhaimi, A. Ammonia flux tailoring on the quality of AlN epilayers grown by pulsed atomic-layer epitaxy techniques on (0001)-oriented sapphire substrates via MOCVD. CrystEngComm 2019, 21, 2009-2017. [CrossRef]

24. Kum, D.; Byun, D. The Effect of Substrate Surface Roughness on GaN Growth Using MOCVD Process. J. Electron. Mater. 1997, 26, 1098-1102. [CrossRef]

25. Guillaume, G.; Gael, G.; Marc, P.; Eric, F.; Daniel, A.; Yvon, C.; Favrice, S. A detailed study of AlN and GaN grown on Silicon-on-porous Silicon substrate. Phys. Status Solidi A 2017, 214, 1600450.

26. Wang, K.; Yu, T.; Wei, Y.; Li, M.; Zhang, G.; Fan, S. Coordinated stress management and dislocation control in GaN growth on Si (111) substrates by using a carbon nanotube mask. Nanoscale 2019, 11, 4489-4495. [CrossRef]

27. Chen, Y.; Chen, Z.; Li, J.; Chen, Y.; Li, C.; Zhan, J.; Yu, T.; Kang, X.; Jiao, F.; Li, S.; et al. A study of GaN nucleation and coalescence in the initial growth stages on nanoscale patterned sapphire substrates via MOCVD. CrystEngComm 2018, 20, 6811-6820. [CrossRef]

28. Kisielowski, C.; Kruger, J.; Ruvimov, S.; Suski, T.; Ager, J.W.; Jones, E.; Liliental-Weber, Z.; Rubin, M.; Weber, E.R.; Bremser, M.D.; et al. Strain-related phenomena in GaN thin films. Phys. Rev. B 1996, 54, 17745. [CrossRef]

29. Chowdhury, S.; Biswas, D. Impact of varying buffer thickness generated strain and threading dislocations on the formation of plasma assisted MBE grown ultra-thin AlGaN/GaN heterostructure on silicon. AIP Adv. 2015, 5, 057149. [CrossRef]

30. Tanoto, H.; Yoon, S.F.; Loke, W.K.; Chen, K.P.; Fitzgerald, E.A.; Dohrman, C.; Narayanan, B. Heteroepitaxial growth of GaAs on (100) Ge/ Si using migration enhanced epitaxy. J. Appl. Phys. 2008, 103, 104901. [CrossRef]

31. Tan, B.; Hu, J.; Zhang, J.; Zhang, Y.; Long, H.; Chen, J.; Du, S.; Dai, J.; Chen, C.; Xu, J.; et al. AlN gradient interlayer design for the growth of high-quality AlN epitaxial film on sputtered AlN/sapphire substrate. CrystEngComm 2018, 20, 6557-6564. [CrossRef]

32. Akyol, F.; Nath, D.; Krishnamoorthy, S.; Park, P.; Rajan, S. Suppression of electron overglow and efficiency droop in N-polar GaN green light emitting diodes. Appl. Phys. Lett. 2012, 100, 111118. [CrossRef]

33. Piprek, J.; Romer, F.; Witzigmann, B. On the uncertainly of the Auger recombination coefficient extracted from InGaN/GaN light-emitting diode efficiency droop measurements. Appl. Phys. Lett. 2015, 106, 101101. [CrossRef] 